\title{
Across the Spectrums
}

\author{
By Jack M. Gorman, MD
}

Although most issues of CNS Spectrums include several articles based on a central theme, the journal also includes a substantial number of original research and review articles and clinical reports unrelated to the monthly theme. Lately, perhaps in response to the inclusion of CNS Spectrums in Index Medicus (under the citation CNS Spectr) and the resulting increase in its impact factor, we are receiving a steadily increasing stream of unsolicited manuscripts. This is most welcome. Although our peer-review system is a serious one and we cannot guarantee publication, we are eager to receive the best work from scientists and clinicians in neurology, psychiatry, and related fields regardless of the subject matter.

This month's issue includes recently submitted and accepted articles, none of which revolve around a particular theme, but all of which are informative and important.

Roy Freeman, MD, notes that pain is the source of a great deal of suffering among patients with peripheral neuropathy of various forms. Much is now understood about the basic neuronal changes that are responsible for this type of chronic and often debilitating pain, and this knowledge is guiding new approaches to treatment. So-called "adjuvant analgesics," medications from a variety of classes not generally thought of as pain killers, are becoming standards in the treatment of neuropathic pain. Freeman reviews the use of these treatments, concluding that careful attention to a patient's level of suffering should enable the physician to design a pharmacologic intervention that will alleviate symptoms.

Aggression in children referred for psychiatric care is the subject of a careful original research study conducted and reported by Kara Zivin Bambauer, $\mathrm{PhD}$, and Daniel F. Connor, MD. While controversy swirls around the proper treatment of children with such well-defined illnesses as depression and attentiondeficit disorder, there are few guidelines, controversial or not, for the therapeutic approach toward children whose psychopathology includes aggressive behavior. Bambauer and Connor found that aggression is more common in children referred for psychiatric treatment than in children culled from the community, but that it is essentially a comorbid condition that requires its own attention and treatment approach. Thus, children with a variety of psychiatric problems are likely to also manifest aggressive behavior, just as many adults with psychiatric illness also suffer from substance abuse. The pervading thought in the latter situation has always been that both the presenting condition and the comorbid substance abuse require separate attention. The authors argue that aggressive behavior in a child with a presenting psychiatric illness must be treated in the same way.

The neurobiology of fear has become a major subject of interest among scientists and clinicians because it is relatively easily modeled in animals. We, therefore, have been able to make substantial progress in understanding the genes, proteins, cells, and neural circuits that are responsible for a variety of types of fear. This knowledge has been directly translated into better understanding of humans with "fear-related" conditions. Although anxiety disorders generally are considered the prototype of the "fear-related" disorders, Jonathan D. Huppert, $\mathrm{PhD}$, and Thomas E. Smith, MD, remind us that fear and anxiety are important components of many psychiatric illnesses. In this case, the investigators studied a group of patients with schizophrenia or schizoaffective disorder and found that most of their subjects $(62 \%)$ had at least one comorbid anxiety disorder. Not only does this information help focus clinicians' attention on the need to address serious anxiety symptoms among patients with psychotic disorders, it also raises the question of how what we know about the neurobiology of fear and anxiety may contribute to improved comprehension of the pathophysiology of schizophrenia.

One of the most important decisions a physician has to make is the choice of a specific medication when several choices in a single category are available. This is clearly the case with respect to depression, for which there are now a multitude of antidepressants available from the "post-Prozac" era. Some of these medications are now generic and, therefore, relatively inexpensive. Stephen M. Stahl, MD, PhD, and colleagues tackle the complex issue of whether dual-reuptake inhibitors, antidepressants that affect presynaptic reuptake of both norepinephrine and serotonin, have any advantage over selective serotonin reuptake inhibitors. He

Dr. Gorman is the editor of this journal and professor of psychiatry and professor of neuroscience in the Department of Psychiatry at Mount Sinai School of Medicine in New York City. 
also discusses the relative benefits among the three dual-reuptake inhibitors, two of which are approved for use in the United States. These are tricky areas because the data are limited and what is available is mostly proprietary. The review by Stahl and colleagues is nevertheless extremely balanced and informative and should be a significant help to clinicians treating patients with depression.

Finally, the article by Gabriella R. Kellerman, $\mathrm{BA}$, and colleagues, is an example of one of the many delights I have as editor of CNS Spectrums. Frequently, a bright medical student interested in psychiatry, neurology, or neuroscience will approach me with a research idea. I generally tell them that the first step is to review the literature on the topic to see what is already known and what studies have already been conducted. Occasionally, the product of that literature search is a fascinating paper that I believe others will be interested in and, therefore, recommend for submission to CNS Spectrums. Because I am involved in the authorship of such papers, I have no further decision-making capacity. Therefore, it is fully possible that CNS Spectrums will reject a paper on which I am a co-author. In this case, however, Kellerman, Jin Fan, $\mathrm{PhD}$, and I were successful with an article detailing the literature on auditory abnormalities in patients with autism. We hope this review will prove useful for those interested in autism and will suggest further research studies in this area.

And so this month, we have articles in psychiatry and neurology, representing original research and reviews, by established people in our field as well as newcomers. All in all, this diversity represents exactly what CNS Spectrums strives to represent. CNS

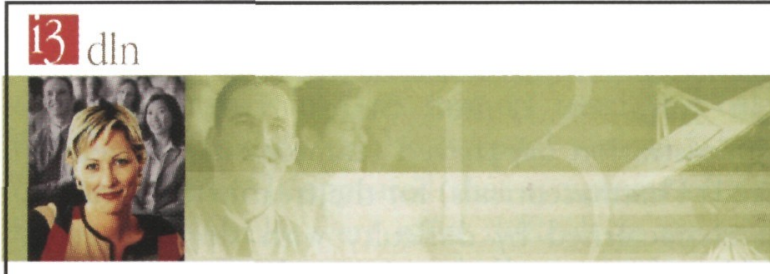

Free CME Video and Web Cast

\section{Bipolar Disorder: What's New in Mood} Stabilization for Mania and Mixed States?

Presenters:

Terence A. Ketter, MD

Professor of Psychiatry \&

Behavioral Sciences

Chief, Bipolar Disorders Clinic

Stanford University School of

Medicine

Richard Weisler, MD

Adjunct Professor of Psychiatry University of North Carolina School of

Medicine, Chapel Hill

Adjunct Assistant Professor of

Psychiatry and Behavioral Sciences

Duke University Medical Center

Moderator:

Henry Nasrallah, MD

Associate Dean

Professor of Psychiatry, Neurology and Neuroscience

University of Cincinnati Medical Center

Learning Objectives:

At the end of this educational activity, participants should be able to:

1. Describe various currently-approved treatments for bipolar disorder, including mood stabilizers and atypical antipsychotics

2. Describe the efficacy, safety and tolerability of extended-release capsules of carbamazepine, the newest FDA-approved mood stabilizer for mania and mixed states

3. Formulate a pharmacologic treatment plan for the patient with acute bipolar mania, including manic and mixed episodes and propose a management strategy to achieve enduring stability

Statement of Need

Bipolar Disorder is a serious neuropsychiatric illness, with an estimated prevalence of $3.7 \%$ [over 10 million Americans]. Bipolar disorder is characterized by recurrent episodes of mania, dysphoric mania or depression, with periods of euthymic mood in between episodes. The disorder can have devastating effects on an individual's life, although proper diagnosis and earlier treatment can usually alter the course of the illness. The atypical antipsychotics have proven effective to treat bipolar mania, but are challenged by metabolic side effects, such as obesity, diabetes and dyslipidemia, and also other side-effects such as sexual dysfunction and movement disorders. i3 DLN faculty members will discuss the efficacy and safety profiles of all the treatment options for bipolar disorder including the data of the FDA-controlled trials of the most recently approved mood stabilizer, extended release carbamazepine and compare it to existing treatments. The unmet needs of bipolar disorder will also be discussed.

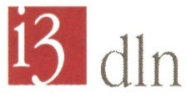

(4) UNIVERSITY OF

This activity is co-sponsored by i3 DLN and The University of Florida College of Pharmacy.

This activity is designed for physicians, pharmacists, nurses, psychologists and case managers.

For more information on this or other i3 DLN CME activities, visit www.i3din.com or call 1-800-326-9166.

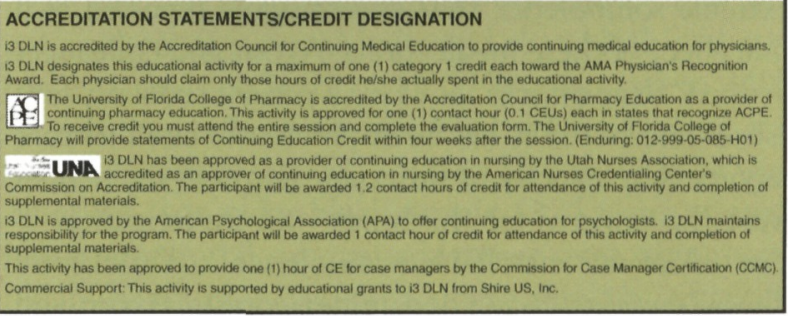

